\title{
THE EFFECT OF THE INITIAL LAYER ON THE REVERSAL MECHANISM IN COCR FILMS
}

\section{COCK LODDER AND LI CHENG-ZHANG*}

University of Twente, P. O. Box 217, 7500 AE Enschede, The Netherlands *Institute of Computing Technology, P.0. Box 2705, Beijing, China

ABSTRACT: It is well known that the initial layer of sputtered $\mathrm{CoCr}$ films can have quite different magnetic properties compared with the succeeding growth of the perpendicular anisotroplc "top layer". To understand the influence of the initiallayer on the magnetic behaviour we have measured the dependence of $\mathrm{OR}$, Hc and hysteresis loss as functions of the angle with the applied field (Ha) as a parameter [5]. In this paper we report that with increasing Hc $\perp / H k$ values the orientation direction of the magnetization gradually changes from in-plane $(\mathrm{OR} \perp=0.7)$ to perpendicular $(\mathrm{OR}=3.9)$. The angular dependece of $\mathrm{Hc}$ is closely related to the value of $\mathrm{Ha}$. It was found that $\mathrm{Hc} / \mathrm{HcL}$ drastically decreases at $\mathrm{Ha}=\mathrm{Hc} \perp \max (159 \mathrm{kA} / \mathrm{m})$ with decreasing $\mathrm{Ha}$. The change of amplitude for $\mathrm{H} / /$ is much smaller than for $\mathrm{HC}$ therefore we introduce a critical field $\mathrm{HcO}$ where $\mathrm{HcL}=\mathrm{Hc} / /$. If $\mathrm{Ha}$ is changed from $\mathrm{Ha}>\mathrm{HcO}$ to $\mathrm{Ha}<\mathrm{HcO}$ the magnetic behaviour will be changed from a typical perpendicular to an in-plane anisotropy. From this we can assume that in the high fleld range( $\mathrm{Ha}>\mathrm{HCO}$ ) the $\mathrm{Hc}$ of $\mathrm{CoCr}$ mainly results from the perpendicular"top layer", but in the low field range Ho basically depends on the behaviour of the initial layer having in-plane anisotropy. If $\mathrm{Ha}>\mathrm{HcO}$ the $\mathrm{Hc} / \mathrm{Hc} / /$ decreases monotonically with the angle and for $\mathrm{Ha}=\mathrm{Hcl} \max$ the $\mathrm{Hc} / \mathrm{Hc} / /$ vs $\theta$ curve follows approximately the calculated inverse $\operatorname{Cos}(90-\theta)$ line. However, for $\mathrm{Ha}=\mathrm{Hco}(=80 \mathrm{kA} / \mathrm{m})$ the measured curves deviate from this calculated one. This can explained by measuring the normalized demagnetizingfactor $N$ vs. $\theta$ curve. For several films we found that if $\theta<30$, there is a sharp increase of $\mathrm{N}$.

\section{INTRODUCTION}

It is generally agreed that sputtered $\mathrm{CoCr}$ films having perpendicular anisotropy are in a compositionally segregated state $[1,12]$ and have a typical columnar microstructure [7]. Recently it was discovered from a lot of experimental data $[1,7,9]$ that the initial layer shows a quite different crystalline orientation and magnetic behaviour, compared with the succeeding growth of a perpendicular anisotropic "top layer". The initial layer can exhibit a significantly different orientation of the easy axis from a random to a rather good in-plane orientation, depending on various deposition conditions. In general, the coercivity of the initial layer is much less than that of the "top layer". It can be expected that the initial layer will exert a tremendous influence on the magnetization reversal mechanism of $\mathrm{CoCr}$ films. It was proposed that the magnetization reversal process in an initial layer seems to take place exclusively through the in-plane domain-wall motion $[2,3]$, and the presence of an in-plane magnetization component is favourable for forming stripe-domain configurations of $\mathrm{CoCr}$ films[5]. Therefore, the reversal mechanism is complicated by the contributions from both incoherent rotation with perpendicular orientation of the magnetization (top layer) and domain-wall motion with either in-plane or randon orientation of the magnetization (initial layer). In order to investigate the precise magnetization process, this paper reports on the dependence of OR, coercivity and hysteresis loss of $\mathrm{COCr}$ films on the angle, which is the angle between the applied field and the film normal, with the applied field as parameter. In addition, the influence of the demagnetizing field on the angular dependence of the magnetic behaviour in $\mathrm{CoCr}$ films is also examined.

\section{EXPERIMENTAL PROCEDURE}

The $\mathrm{CoCr}$ films are RF magnetron sputtered on silicon and $\mathrm{RF}$ sputtered on polyimide (no:6) substrates. For the latter $\Delta \theta_{50}=9 \sim 10$. X-ray fluoresence was used to check the and numeral integrals were used to calculate the hysteresis and numeral integrals were used to calculate the hysteresis
loss of these films. We paid particular attention to investigating a typical CoCrfilm (no, 6), which has a very high coercivity $(159 \mathrm{kA} / \mathrm{m})$ with a quite obvious in-plane magnetization component, because it is very advantageous to study the relation between the microstructure and magnetization reversal mechanism of $\mathrm{CoCr} f i l m s$. In order to compare the experimental data with the calculated curve of the domain-wall motion, a pure nickel specimen was also examined. The related magnetic propertles obtained from the VSM and Torque Magnetometer are summarized in table 1.

\begin{tabular}{|c|c|c|c|c|c|c|c|}
\hline No & $\frac{\text { Table 1: }}{\substack{\mathrm{nm} \\
\text { thickness }}}$ & $\frac{\mathrm{Ms}}{\mathrm{MA} / \mathrm{m}}$ & $\begin{array}{r}\mathrm{Hc} \perp \\
\mathrm{KA} / \mathrm{m}\end{array}$ & $\begin{array}{c}\mathrm{HC} \perp / \mathrm{Hk} \\
\%\end{array}$ & Rs $(0)$ & $\overline{\mathrm{O}} \mathrm{R}$ & \begin{tabular}{|c|} 
Wh1 \\
$\mathrm{J} / \mathrm{m} * 10^{\circ}$
\end{tabular} \\
\hline 1 & 45 & 334 & 35 & 1.78 & 0 . & 0.7 & 1.21 \\
\hline 2 & 1230 & 424 & 15 & 9 & 0 . & 0.75 & 1.43 \\
\hline 3 & 650 & 46 & 35.0 & 5.65 & 0. & 2.6 & 4.4 \\
\hline 4 & 1220 & 468 & 46.2 & 7.5 & 0.1 & 3.9 & 5.5 \\
\hline 5 & 650 & 321 & 73.2 & 14.1 & 0.212 & 3.6 & 6.0 \\
\hline 6 & 265 & 467 & 159.2 & 29.8 & 0.3 & 2.6 & 24.2 \\
\hline 7 & 5417 & 484 & 8. 75 & 1.0 & 0.014 & 0.032 & 0.0 \\
\hline
\end{tabular}

The definitions of some parameters used are as follows: 1) The squareness ratio $\operatorname{Rs}(\theta)=\operatorname{Mr}(\theta) / M s$ (uncorrected for the demagnetizing field). 2) Relative orientation ratio $\mathrm{OR}(\theta)=\operatorname{Rs} \perp / \operatorname{Rs}(\theta) .3)$ The measured coercivity at $800 \mathrm{kA} / \mathrm{m}$ is considered to be the film's coercivity Hc $\perp$ max. Due to the influence of the demagnetlzing field, the deviation of the magnetization from the film normal will take place by varying degrees. The larger the Rs(0)the less the the deviation of magnetization from the easy axis of a $\mathrm{CoCr} f i l m$ in the remanent state will be. Therefore, in a certain sense the amplitude of Rs(O) reflects the ability of CoCr films to resist or weaken the influence of the demagnetizing field. Due to the influence of the columnar microstructure and perpendicular anisotropy, the $\mathrm{N} \downarrow$ factor of $\mathrm{CoCr}$ films (the demagnetizing factor along the film normal direction) is not necessarily equal to 1 . Sometimes, the $N_{\perp}$ is much less than 1. In order to simplify matters, all the demagnetizing factors $\mathrm{N}$ were normalized by those at $0^{\circ}$.

\section{EXPERIMENTAL RESULTS AND DISCUSSION}

The results listed in table 1 show that as the Hcl/Hk values of $\mathrm{CoCr} f 11 \mathrm{~ms}$ increase, all the Rs(0), OR and $W \perp$ values also increase almost monotonically. With increasing $\mathrm{Hcl} / \mathrm{Hk}$, the orientation direction of the magnetization gradually changes from the in-plane direction $(\mathrm{OR} \perp=0.7)$ to the changes from the in-plane direction $(\mathrm{OR} \perp=0.7)$ to the
perpendicular direction $(\mathrm{OR} \perp=3.9)$. This means the degree of orientation of themagnetization along the perpendicular direction increases with the $\mathrm{Hcl} / \mathrm{Hk}$. At the same time with increasing $\mathrm{Hc} \perp / \mathrm{Hk}$, the absolute values of $\mathrm{Rs}(\mathrm{O})$ increase from 0.03 to 0.39 . This fact clearly shows that the higher the $\mathrm{Hcl} / \mathrm{Hk}$, the less the influence of the demagnetizing field on the magnetization orientation will be, so that a $\mathrm{CoCr} \mathrm{film}$ with a high $\mathrm{Hcl} / \mathrm{Hk}$ is advantageous for improving the stability of magnetic recording media. This is supported by the following arguments: for a $\mathrm{CoCr}$ magnetic disk the reproduced signal $\mathrm{Ep}$ is in direct proportion to $\mathrm{HCl}$ and the recording density DSO is in inverse proportion to HcL[10]. it was known that for a $\mathrm{CoCr}_{\mathrm{f}} \mathrm{film}$ the magnetization reversal mechanism strongly depends on the amplitude and direction of the applied field [5], so that the angular dependeces of $\mathrm{Hc}(\theta) / \mathrm{Hcl}, \mathrm{OR}(\theta)$ and $\mathrm{Wh}(\theta) /$ Wh with the field as a parameter were measured. In order to have a thoroughinvestigation of the magnetization behaviour of CoCr films, a $265 \mathrm{~nm}$ specimen (no.6), which has a very high coercivity $(\mathrm{Hc}=159 \mathrm{KA} / \mathrm{m})$, was chosen.

Orientation ratio, hysteresis loss and critical coercivity The angular dependence of $\mathrm{OR}(\theta)$ with the field as parameter is shown in Fig. 1. It is very interesting to see that when the applied field is higher than $\mathrm{Hclmax}(159 \mathrm{kA} / \mathrm{m})$, the behaviour of the $\mathrm{OR}$ vs. $\theta$ curves is that usually seen for perpendicular oriented media, but in the low field range

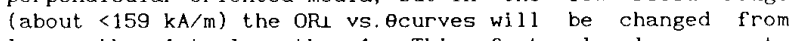
larger than 1 to less than 1 . This fact clearly suggests that the orientation direction of the magnetization will be changed from the perpendicular to the in-plane direction if Ha<Hcimax. It is clearly shown from Fig.2 that Hcl drastically decreases at Ha=Helmax $(159 \mathrm{kA} / \mathrm{m})$ with 
decreasing applled fleld. Although both $\mathrm{HCl}$ and $\mathrm{HC} / /$ decrease with decreasing field, the change in magnitude of $\mathrm{Hc} \perp$ is much larger than that of $\mathrm{Hc} / /$. If $\mathrm{Ha}=\mathrm{HcO}(80 \mathrm{kA} / \mathrm{m})$, the Hcs is just equal to $\mathrm{Hc} / /$. Hereafter this coercivity Hco is referred to as the critical coercivity. As the applied field is changed from Ha>Hco to Ha<Hco the magnetic behaviour of a CoCr fllm will be changed from a typical perpendicular anisotropic one to an in-plane anisotropic media. It is because the $\mathrm{HcL} / \mathrm{HC} / /$ of $a \mathrm{CoCr} \mathrm{film}$ is larger or smaller than 1 that this indicates whether the film has the charateristics of perpendicular or in-plane anisotropy. Perhaps it is reasonable from this result to assume that in the high field range (Ha>Hco) the coercivity of this type of $\mathrm{CoCr}$ films mainly results from the "top Layer" of $\mathrm{CoCr}$ having perpendicular anisotropy, but in the low field range the coercivity basically depends on the behaviour of the initial layer having in-plane anisotropy. In addition, if $\mathrm{Ha}<\mathrm{HcO}$, the absolute value of $\mathrm{HcL}$ will
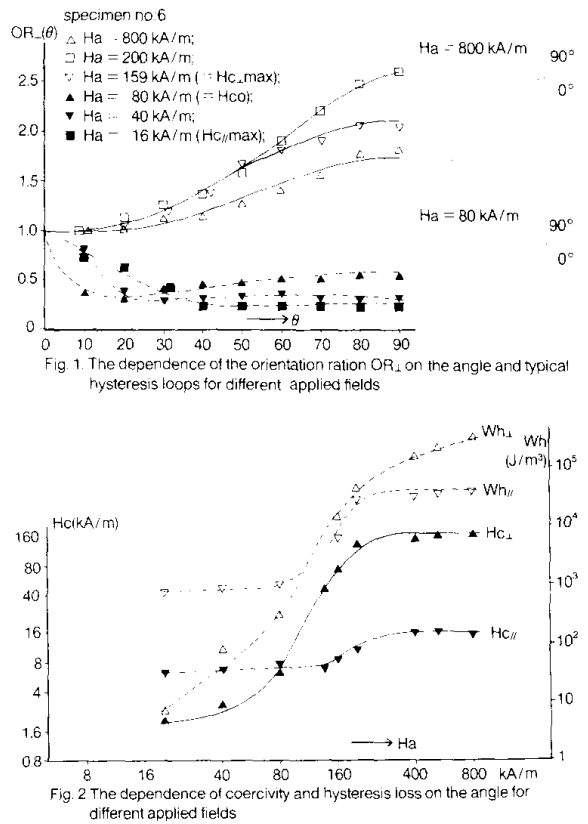

drastically decrease from $159 \mathrm{kA} / \mathrm{m}$ to a very small value of about $3 \mathrm{kA} / \mathrm{m}$, but the $\mathrm{Hc} / /$ only reduces from $16 \mathrm{kA} / \mathrm{m}$ to 7.2 $\mathrm{kA} / \mathrm{m}$. It is noticeable that this value is almost identical with the coercivity of low $\mathrm{Hc} / \mathrm{Hk} \mathrm{films}(8 \mathrm{kA} / \mathrm{m})$ and similar values of the initial layer [9]. It can be also seen from Fig. 1 that the hysteresis loop of this specimen has a typical characteristic of perpendicular anisotropy if Ha> Hco. However, if the applied field is lower than the critical coercivity, it seems that the main perpendicular oriented layer no longer switches. Only the in-plane oriented layer with low coercivity (about $8 \mathrm{KA} / \mathrm{m}$ ) can respond to the applied field. At this time its hysteresis loop becomes a typical one of in-plane anisotropy. Therefore, it could be reasonable to assume that the magnetization reversal for this "top layer" has rather sharp switching properties, in the low applied field the magnetic behaviour seems to only depend on the initial layer. According to the amplitude of the discontonuous magnetization jump in the in-plane hysteresis $100 \mathrm{p}$, the thickness of this in-plane oriented layer is roughly estimated at about 50nm [4].

The dependences of WhL and Wh// on the applied field, as seen in Fig. 2, reveal that: 1) if the applied field is higher than $\mathrm{Hco}$, the $\mathrm{CoCr}$ film exhibits a typical perpendicularly anisotropic behaviour ( $W h /$ Wh $>1)$. The angular dependence of wh/Wh t monotonically decrease with increasing $\theta$, which basically follows the change tendency of incoherent rotation reversal [6]. However, this film wil] become an in-plane oriented media (Wh1/Wh//<1) if Ha<Hco. The change pattern obviously deviates from a straight line, which considered as an important indication of domain-wall motion [6]. The reason to cause such discrepency have been described elsewhere. 2) Although the Whi and wh// decrease monotonically with decreasing $\mathrm{Ha}$, the change in magnitude of
Whi is far larger than that of Wh//. As $\mathrm{Ha}$ decreases from 800 to $16 \mathrm{kA} / \mathrm{m}$, Wh// decrease by a factor of $5.3 * 10^{2}$, but the decrease in amplitude of WhL is as high as a factor of 5. 1*10 $10^{4}$. Especially in the field ranges between $\mathrm{HCl}$ max and Hco a small change in amplitude of $\mathrm{Ha}$ can cause the hysteresis loss to change enormously. Therefore, in order to ensure that the CoCr films exhibit a stable perpendicular property $\mathrm{Ha}>\mathrm{Hcl}$ max is an indispensable condition.

Relative in-plane coercivity.

All the above results suggest that, as a whole, this $\mathrm{CoCr}$ film has strong perpendicular anisotropy and consists of two quite different layers, one a typical main perpendicularly oriented top layer, the other an in-plane oriented layer with rather low coercivity. It is reasonable to infer that the response of the whole $f i l m$ can be considered as the superposition of the response of each layer to the applied field. In order to investigate the correlation between the magnetization reversal mechanism of $\mathrm{CoCr} f 11 \mathrm{~m}$ and $\mathrm{Ha}$, the magnetization rever seen from this figure the effect of the applied field on the angular dependence of the coercivity is quite remarkable. If the applied field is higher than the HcLmax $(159 \mathrm{kA} / \mathrm{m})$, the $\mathrm{Hc} / \mathrm{Hc} / / \mathrm{vs} . \theta$ curves decrease monotonically with increasing angle. This behaviour is qualitatively consistent with the characteristic of the incoherent rotation $\cos \theta$ law [5]. As the applled field is cont inuously decreased and approaches

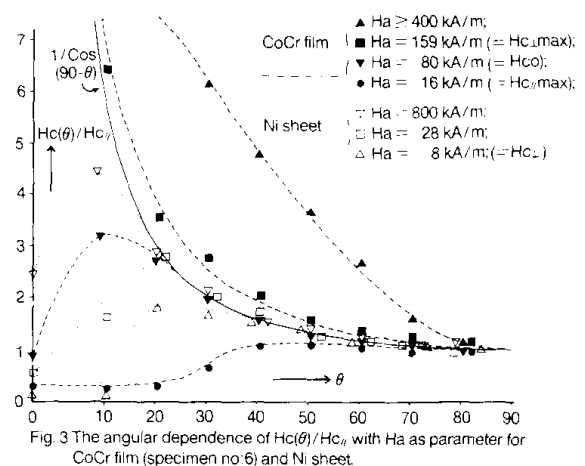

Hcl max, the experimental form of the relative coercivity will gradually change from following the $\operatorname{Cos} \theta$ to the inverse $\operatorname{Cos}(90-\theta)$ law. It is well known [11] that the form of $\mathrm{Hc} / \mathrm{Hc} / /$ will follow the inverse Cos law if its magnetization is reversed by domain-wall motion. However, if $\mathrm{Ha}$ is further decreased, becoming lower than Hco, the form of $\mathrm{Hc} / \mathrm{Hc} / /$ will again deviates from the inverse Cos law. It can be easily seen from Fig. 3 that at different Ha for each $\mathrm{Hc} / \mathrm{Hc} / /$ vs. $\theta$ curve there is a critical angle $\theta \mathrm{cr}$ above which the form of $\mathrm{Hc} / \mathrm{Hc} / /$ is almost Identical with the inverse Cos curve, but below this angle the $\mathrm{Hc} / \mathrm{Hc} / /$ drastically decreases with a decreasing angle. With decreasing Ha, this angle is gradually moved towards a higher value.

Normalized demagnetizlng factor.

In order to clarify the physical meaning of the critical angle, the normalized demagnetizing factor $N$ vs $\theta$ curves are shown in Fig. 4. for specimen no:6. If the angle is below

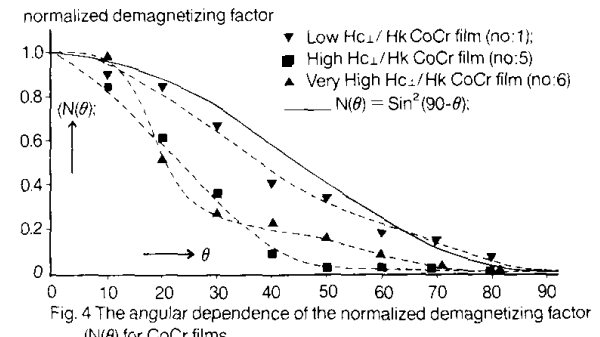
$(N(\theta)$ for CoCr films

about $30^{\circ}$, the dernagnetizing factor sharply increases. On the contrary, if the angle is changed to approach the in-plane direction, it only causes $\mathrm{N}$ to increase slightly. Therefore, it may be inferred that if the direction of $\mathrm{Ha}$ is very close to the film normal, the internal field will become so small that the working point of the magnetization 
will move to the declining minor loop in the field range below Hco. Hence it results in a sharp decrease of $\mathrm{Hc} / \mathrm{Hc} / /$. According to the same viewpoint, it is easily understood why the shape of the ORI vs. $\theta$ curves in the field range of $\mathrm{Ha}<\mathrm{HCO}$ (seen in Fig. 1 ) is similar to the variation of the coercivity, if the angle is decreased, starting from the in-plane direction. At first ORs decreases very slowly, but once the angle is lower than some of the critical angles, the ORI value will increase sharply and the critical angle will gradually move to a larger angle with decreasing field. For isotropic recording films the angular dependence of the normalized demagnetizing factor follows the $N(\theta)=\operatorname{Sin}^{2}(90-\theta)$ [13]. Comparing the calculated curve drawn by the solid line with the measured ones for $\mathrm{CoC} r$ films, it is clearly seen from fig. 4 that for low coerclvity films the $N$ vs. $\theta$ curve is quite consistent with the calculated one. This fact suggests that the characteristic of this $111 \mathrm{~m}$ approaches that of the isotropic recording media. This argument is supported by that for low coercivity films; their several magnetic parameters (such as $\mathrm{OR}$, Hc etc.) are almost independent of the angle [5]. However, as the $\mathrm{Hc} 1 / \mathrm{Hk}$ value of $\mathrm{CoCr} f i l m s$ increases, the deviation of the $N$ vs. $\theta$ curves from the calculated one will gradually increase.

Relat ive hysteresis loss.

The dependence of the measured hysteresis loss for specimen 6 is plotted gainst different $\mathrm{Ha}$ in Figs.5a,b, representing the applied field respectively higher and lower than the Hcimax of $159 \mathrm{KA} / \mathrm{m}$. It can be seen from Fig. 5a that he hysteresis loss Wh/Wh 1 vs. $\theta$ curves for four different fields without of exception decrease monotonically with increasing angle, and qualitatively follow the same change pattern expected by the incoherent rotation models in flelds above $159 \mathrm{kA} / \mathrm{m}$. However, if Hal is continuously decreased to be below this, the hysteresis loss Wh/Wh 1 vs. $\theta$ curves follow quite different variations. If the angle increases, the hysteresis loss first increases, reaching a maximum value at an appropriate critical angle; further increase of the angle results in a diminished hysteresls loss. This fact suggests that at present the magnetization reversal mechanism of $\mathrm{CoCr}$ films must differ from that of the rotation reversal model.

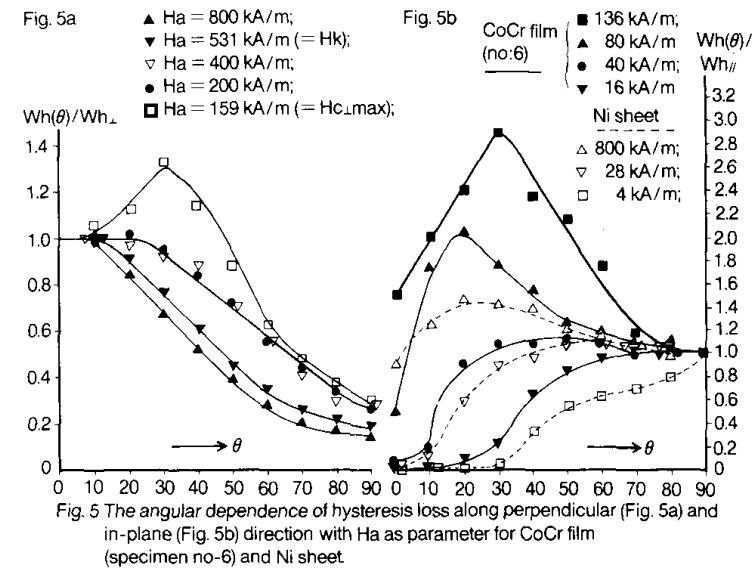

In order to present a more clear image the angular dependence of the hysteresis loss $W h / W h / /$ with respect to different fields is also shown in Fig. 5b, because the changed from the fllm normal to an in-plane direction in the changed from the fllm normal to an in-plane direction in the
field below $159 \mathrm{kA} / \mathrm{m}$. According to previous analyses of $\mathrm{Hc} / \mathrm{Hc} / /$ behaviour for $\mathrm{CoCr}$ fllms, we have learnt that if $\mathrm{Ha}$ is Jower than $159 \mathrm{KA} / \mathrm{m}$, the magnetization reversal occurs by domaln-wall motion and if this is so the characteristics of the hysteresis loss should be independent of the angle, but a very drastic angular dependence of the measured hysteresis loss appears instead of a stralght 1 ine. In order to explain the relatively large discrepancy between the experimental and theoretical results based on the domain-wall displacement, a pure nickel sheet was also examined because it is well known that its magnetization reversal mechanism belongs to domain-wall motion and the magnetization aligns in the in-plane direction. Compared with the angular depedencies of hysteresis loss $\mathrm{Wh} / \mathrm{Wh} / /$ drawn with a dashed linefor the nickel specimen, it is clear that the angular dependencies of $\mathrm{Wh} / \mathrm{Wh} / /$ for $\mathrm{CoCr}$ films is strikingly similar to the behaviour of the nickel specimen. As the applied fleld decreases, the critical angle, which corresponds to the the maximum hysteresis loss $\mathrm{Wh} / \mathrm{Wh} / /$, gradually moves to a larger angle. It is shown in Fig. $5 \mathrm{~b}$ that above this critical angle, the $\mathrm{wh} / \mathrm{Wh} / / \mathrm{vs}$. $\theta$ curves ascend in varying degrees with decreasing angle, starting from the in-plane direction, and below it descend drastically with decreasing angle. This latter behaviour is due to the strong influence of the demagnetizing fleld. In the presence of the demagnetlizing field the Wh/Wh// vs. $\theta$ curves no longer follow the stralght-line law for a thin film having in-plane anisotropy. In view of the same consideration, the $\mathrm{Hc} / \mathrm{Hc} / /$ vs. $\theta$ curves for both the nickel specimens drawn with dashed lines ( $\mathrm{Ha}$ ranging from 8 to $800 \mathrm{KA} / \mathrm{m}$ ) and the $\mathrm{CoCr}$ film (no.6, in the field range below $159 \mathrm{KA} / \mathrm{m}$ ) are shown in $\mathrm{Fig}$ 3. The change tendencles of the $\mathrm{Hc} / \mathrm{Hc} / /$ vs. $\theta$ curves again show that there is a striking similarity between the two specimens. Above the critical angle the angular dependences almost coinclde with the inverse $\operatorname{Cos}(90-\theta)$ curve, which is thought to be a strong indication of domaln-wall motion. Therefore, it is perhaps reasonable to conclude that in the low field range $\mathrm{Ha}<\mathrm{Hco}$ a $\mathrm{CoCr}$ film presents an in-plane orientation of the magnetization and its magnetization is reversed by in-plane domain-wall motion. In the vicinity of the film normal ( $<$ about $30^{\circ}$ ) the angular dependence for both $\mathrm{Wh} / \mathrm{Wh} / /$ and $\mathrm{Hc} / \mathrm{Hc} / /$ are strongly influenced by the demagnetizing fleld. It is very interesting that a similar behaviour of $\mathrm{Hc} / \mathrm{Hc} / /$ and hysteresis loss also appears in the bulk $\mathrm{CoCr}$ sample (its $\mathrm{Hc}$ is about $1.3 \mathrm{kA} / \mathrm{m}$ ) [8].

\section{CONCLUSIONS}

1) This type of $\mathrm{CoCr}$ films are composed of an initial layer with a succeedingly grown perpendicularly anisotropic "top layer". The magnetization reversal mechanism of $\mathrm{CoCr}$ films consisting malnly of an initial layer is controlled by domain-wall motion and its orientation of the magnetization aligns along the in-plane direction. However, these films exhibit a strong perpendicular anisotropy as a whole and the incoherent rotation reversal dominates. The response of the $\mathrm{CoCr}$ films can be qualitatively interpreted as the superposition of the response of each layer to the applied fleld. 2) The magnetization reversal mechanism strongly depends on the amplitude and direction of the sield. If the applied fleld is larger than Hacl max, the Incoherent rotation process dominates. In the low fleld ranges below it, the magnetization reversal is controlled by the in-plane domain-wall motion. 3) In the range of low $\mathrm{Ha}$ the demagnetizing field exerts a tremendous influence on the magnetic behaviour of $\mathrm{CoCr}$ films, especially in the vicinity of the film normal direction. 4) With increasing $\mathrm{Hcl} / \mathrm{Hk}$ values, the degree of magnetization orientation along the the film normal will increase. The higher the coercivity. the more pronounced the perpendicularly anisotropic characteristlc. According to the angular dependence of $O R$, $\mathrm{Hc}$ and the demagnetizing factor for low $\mathrm{Hc} \perp / \mathrm{Hk} \mathrm{CoCr}$ films the magnetic behaviour approaches an isotropic recording medlum.

\section{ACKNOWLEDGEMENTS}

The authors would Iike to thank Prof.Dr. S. Iwasaki and Dr. K. Ouchi (Tohuku Univ. Japan) for providing specimen 6 . We also appreciate the continued help and support of this work by Prof. Th. J.A. Popma and Messrs. J.D. Baxter and T. Bolhuis.

\section{REFERENCES}

1) H. Hoffmann, IEEE Trans. on Mag-Vol 22, no. 5, 1986

2) C. Byun, J.M.Sivertsen and J.H.Judy, IEEE Trans. Mag-Vol 22, no. 5,1986

3) E.R.Wuor1, J.H. Judy, IEEE Trans. Mag-Vol 20, no. 5, 1984.

4) D.S. Bloomberg, J.A. Phys. Vol 57, no. 1, 1985.

5) Li Cheng-Zhang, J.C. Lodder, IEEE Trans Vol-23 no: 3 (1987)

6) K. Ouch1, S. Iwasak1, IEEE Trans. Vol Mag-23, no: 11987.

7) J.C.Lodder, T. Wielenga and J. Worst, Thin Solid Films, Vol 101, P.61, 1983

8) Li Cheng-Zhang and J.C.Lodder, to be published.

9) C. Byun, J.H.Judy, IEEE Trans, Vol Mag-21, no. 5, 1985

10)Y. Nakamura, S. Yamamoto and S. I wasaki IEEE Trans. Mag Vol 22 no. 5, 1986

11)E. Kondorsky, J. Phys. USSR, Vol 2, 161, 1940

12) J. Meada et al. Japanese J. Appl. Phys. 25(1986) L668.

13) R. M. Josephs, D.S.Crompton and C.S. Crafft, IEEE Trans. Vol Mag-22, no: 5 (1986). 